\title{
Influence of Lateral Field on the Relaxation Oscillation Frequency of Semiconductor Lasers
}

\author{
S. F. Yu and E. Herbert Li, Senior Member, IEEE
}

\begin{abstract}
We demonstrate theoretically that the lateral field distribution can be utilized to enhance the relaxation oscillation frequency of semiconductor lasers. It is found, for some laser parameters, that gain-guided semiconductor lasers with narrow stripe can exhibit higher relaxation oscillation frequency than index-guided devices.
\end{abstract}

\section{INTRODUCTION}

$\mathbf{T}$ THE LATERAL field guiding performance of semiconductor lasers with gain and index guided structures have been studied extensively [1], [2]. Most of the works are concentrated on the analysis of stable and single lateral mode operation under high injection current as well as the uniformity of light-current characteristic [1], [2]. However, the relative important quantity, the relaxation oscillation frequency, under the influence of various optical confinement structures has not been investigated. In this paper, the effects of lateral optical field on the dynamic response of semiconductor lasers is analyzed theoretically.

\section{THEORETICAL ANALYSIS}

The relaxation oscillation frequency of semiconductor lasers including the influence of lateral field can be derived from the modified rate equations model given below

$$
\begin{aligned}
& \frac{d N(t)}{d t}=\frac{I(t)}{q V}-v_{g} \Gamma(N) G(N) P(t)-\frac{N(t)}{\tau_{s}} \\
& \frac{d P(t)}{d t}=v_{g} \Gamma(N) G(N) P(t)-\frac{K N(t)}{\tau_{s}}-\frac{P(t)}{\tau_{p}}
\end{aligned}
$$

where $N$ and $S$ are the equivalent carrier concentration and photon density. $G$ is the equivalent modal gain, $\Gamma$ is the lateral confinement factor, $\tau_{s}$ is the carrier lifetime and $\tau_{p}$ is the photon lifetime. $K$ is the spontaneous emission factor, $I$ is the injection current and $V$ is the volume of waveguide. In (1) and (2), $N, P$ and $\Gamma$ are time dependent. The frequency response of lasers can be obtained by setting,

$$
\begin{gathered}
N(t)=N_{s}+\Delta N(t) \\
P(t)=P_{s}+\Delta P(t)
\end{gathered}
$$

where $s$ stand for steady state. By substituting (3) into (1) and (2), with the assumptions $N_{s} \gg \Delta N$ and $S_{s} \gg \Delta S$, a secondorder differential equation describing a damping harmonic

\footnotetext{
Manuscript received April 5, 1995; revised August 21, 1995. This work was supported in part by HKU CRCG, Account Code 337/062/0023.

The authors are with the Department of Electrical and Electronic Engineering, University of Hong Kong, Pokfulam Road, Hong Kong.

Publisher Item Identifier S 0018-9197(96)00341-7.
}

oscillator is deduced. The corresponding relaxation oscillation frequency, $\omega_{f}$, is given by

$$
\omega_{f}^{2}=\frac{v_{g} P_{s}}{\tau_{p}}\left(\Gamma_{s} \frac{\partial G}{\partial N}+G_{s} \frac{\partial \Gamma}{\partial N}\right)
$$

where we have ignored the spontaneous emission term in the derivation. In addition, we also assume that

$$
\begin{aligned}
& G\left(N_{s}+\Delta N\right)=G_{s}+\frac{\partial G}{\partial N} \Delta N \\
& \Gamma\left(N_{s}+\Delta N\right)=\Gamma_{s}+\frac{\partial \Gamma}{\partial N} \Delta N
\end{aligned}
$$

in the derivation of (4). $\partial \Gamma / \partial N$ is the differential confinement factor and this parameter is affected by the lateral confinement structure of semiconductor lasers such as devices with strong index or gain guided structure.

In strong index guided lasers, optical field is strongly guided along the lateral direction due to the step change in refractive index between the core (active region) and the cladding regions. However, external carrier injected into the active region causes a small reduction of refractive index inside the active region. This is because of the carrier induced index change inside the semiconductor material.

If we assumed that the injection carrier is well confined and its distribution is uniform along the active region. The small change of lateral field, $\Delta E_{p}$, can be deduced analytically by using the perturbation method [3] and is given as

$$
\Delta E_{p}=\frac{1}{\int\left|E_{p}\right|^{2} d y} \sum_{q \neq p} \frac{k_{0}^{2} \int E_{q}^{*} \Delta \varepsilon E_{p} d y}{\beta_{q}^{2}-\beta_{p}^{2}} E_{p}
$$

The small variation of permittivity, $\Delta \varepsilon$, in strong index guided lasers can be approximated by $\Delta \varepsilon \approx 2 n \Delta n$ where $\Delta n$ is the small change of refractive index caused by the external injected carrier and $n$ is the built-in refractive index of waveguide. $E$ and $\beta$ is the electric field and longitudinal propagation coefficient, respectively, at steady state. The subscript $p$ and $q$ are integers stand for the lateral mode number and $k_{0}$ is the free space wavevector. As we can see in (6), $\Delta E_{p}$ reduces with the increase of carrier concentration inside the active region but remains unchanged inside the cladding layers. From the definition of $\Gamma$,

$$
\Gamma=\int_{\text {active }}\left|E_{p}\right|^{2} d y / \int_{\text {cladding }}\left|E_{p}\right|^{2} d y+\int_{\text {active }}\left|E_{p}\right|^{2} d y
$$

$\Gamma$ is also reduced with the increase of carrier concentration and this implies that $\partial \Gamma / \partial N$ is less than zero. Therefore, high 
optical gain in index guided devices is not desired for the reason of reduction of relaxation oscillation frequency through $\partial \Gamma / \partial N$ as indicated in (4).

In gain guided devices, the optical field and carrier concentration profile are affected by the injection carrier distribution along the lateral direction and they are determined by the carrier diffusion rate as well as the width of metal stripe. In addition, the differential gain are also varied between the active and absorption regions. In order to analyze the differential confinement factor defined in (4), we use the following assumptions. 1) For devices with narrow stripe width, it is reasonable to assume uniform lateral distribution of carrier concentration inside the active region and is equal to $N_{1} .2$ ) The profile of lateral carrier distribution inside the absorption regions are identical with the optical field distribution and the corresponding peak carrier concentration is equal to $2 N_{2}$ where $N_{2}$ is the average carrier concentration inside the absorption layers [4]. 3) The carriers diffusion rate between the active and absorption regions are assumed to be proportional to the difference of $N_{1}-N_{2}$ and 4) the differential gain of the active and absorption regions is assumed constant and is equal to $\partial G / \partial N_{1}$ and $\partial G / \partial N_{2}$, respectively.

In gain guided devices, it is noted that the average carrier concentration inside the active $\left(N_{1}\right)$ and absorption region $\left(N_{2}\right)$ are varied out of phase. This is because as $N_{1}$ approach its threshold value, photon density start to generate and is absorbed in the absorption region, hence, $N_{2}$ in absorption region is increased due to the absorption. However, $N_{1}$ is reduced with the stimulated emission [4]. Therefore, the net change of equivalent carrier concentration, $\Delta N$, can be related to the net change of carrier concentration inside the active $\left(\Delta N_{1}\right)$ and absorption $\left(\Delta N_{2}\right)$ regions as

$$
\begin{aligned}
& \Delta N_{1}=N_{1}-N_{s 1}=\Delta N \\
& \Delta N_{2}=N_{2}-N_{s 2}=-\Delta N
\end{aligned}
$$

where $N_{s 1}$ and $N_{s 2}$ are the steady state carrier concentration inside the active and absorption regions, respectively. Hence, the corresponding differential confinement factor can be approximated by

$$
\frac{\partial \Gamma}{\partial N}=\frac{\partial \Gamma}{\partial N_{1}}-\frac{\partial \Gamma}{\partial N_{2}}
$$

For pure gain guided waveguide with low loss and gain, the confinement factor can be expressed as [5]

$$
\Gamma \approx \frac{\beta_{i} / k_{0}-\zeta_{2}}{\zeta_{1}-\zeta_{2}}
$$

where $\beta_{i}$ is the imaginary part of the propagation coefficient. We have defined the complex dielectric permittivity, $\varepsilon_{j}$, of region $j$ as

$$
\varepsilon_{j}=\left(n_{j}+i \zeta_{j}\right)^{2}
$$

where $i=\sqrt{ }-1$, the subscript $j$ stands $j=1$ for the active region and $j=2$ for the absorption region. Differentiate (10) with respect to $N$, we get

$$
\frac{\partial \Gamma}{\partial N}=\frac{\frac{1}{k_{0}}\left(\frac{\partial \beta_{i}}{\partial N_{1}}-\frac{\partial \beta_{i}}{\partial N_{2}}\right)+\left[(1-\Gamma) \frac{\partial \zeta_{2}}{\partial N_{2}}-\Gamma \frac{\partial \zeta_{1}}{\partial N_{1}}\right]}{\zeta_{1}-\zeta_{2}} .
$$

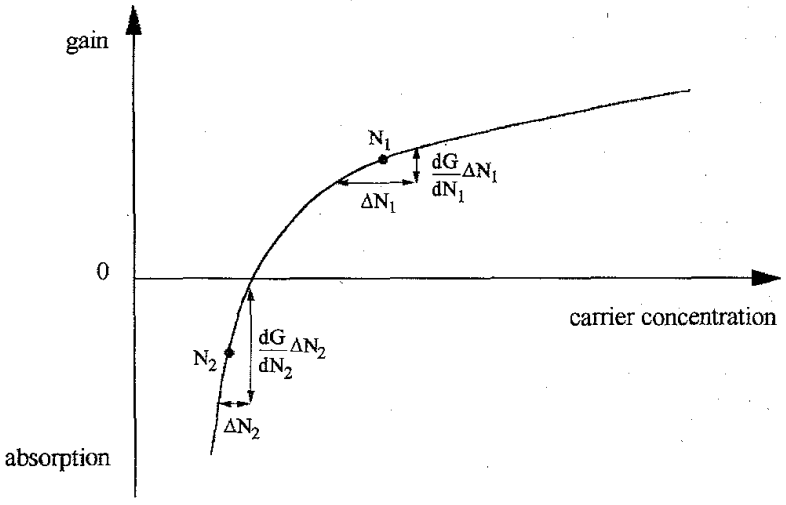

Fig. 1. Optical gain and absorption versus injected carrier density in a semiconductor laser shown schematically. A small change in carrier density affects absorption more strongly than gain.

One of the conditions for mode stability in gain-guided waveguide is $\left(n_{1} \zeta_{1}-n_{2} \zeta_{2}\right)>0$ [5] and this implies that the denominator of (12) should be greater than zero as $n_{1} \sim n_{2}$ and hence $\zeta_{1}>\zeta_{2}$. Furthermore, we should note that $\beta_{i}$ is the net gain of the device and $\beta_{i}$ should increase monotonously with $\Delta N$. Therefore,

$$
\left(\frac{\partial \beta_{i}}{\partial N_{1}}-\frac{\partial \beta_{i}}{\partial N_{2}}\right)=\frac{\partial \beta_{i}}{\partial N} \equiv \frac{\partial G}{\partial N} \geq 0 .
$$

Now, we impose the inequality requirement for the term on the right hand side of (12) enclosed by a square bracket as below

$$
\begin{aligned}
& \frac{(1-\Gamma)}{\Gamma}\left(\frac{\partial \zeta_{2}}{\partial N_{2}} / \frac{\partial \zeta_{1}}{\partial N_{1}}\right) \\
& \quad=\frac{(1-\Gamma) \partial G / \partial N_{2}}{\Gamma \partial G / \partial N_{1}} \geq \mid \text { or } \frac{1-\Gamma}{\Gamma} \geq \frac{\partial G / \partial N_{1}}{\partial G / \partial N_{2}}
\end{aligned}
$$

This inequality holds only for $\Gamma$ is small and $\partial G / \partial N_{1}<$ $\partial G / \partial N_{2}$. In gain guided devices with narrow stripe width (say $3 \mu \mathrm{m}), \Gamma<0.7$ is usually found. In addition, the fundamental nonlinear relation between the optical gain and the injected carrier density in semiconductor lasers indicated that the a small change in carrier density affects absorption more strongly than gain [1]. This relation is shown schematically in Fig. 1. Therefore, with suitable laser parameters, it is possible to achieve the inequality of (14) and gain guided devices can exhibit higher relaxation oscillation frequency than index guided lasers provided than the differential gain of the active and absorption regions and lateral confinement factor satisfy (14).

The numerical value of the differential confinement factor of a gain guided Fabry-Pérot semiconductor laser is also calculated for comparison. The laser model used in the analysis is similar to that given in [4] and the corresponding parameters used in the following analysis are given in Table I. Fig. 2 shows the calculated differential confinement factor $\left(\partial \Gamma / \partial N_{1}\right.$ and $\left.\partial \Gamma / \partial N_{2}\right)$ against $N_{1}-N_{2}$ for device with stripe width equal to 3,4 , and $5 \mu \mathrm{m}$. In this numerical analysis, the value of $\partial \Gamma / \partial N_{1}$ is positive and that of $\partial \Gamma / \partial N_{2}$ is negative. Using (9), the differential confinement factor $\partial \Gamma / \partial N$ is positive for these 


\section{TABLE I}

\begin{tabular}{l|l}
\hline Carrier lifetime & $4 \times 10^{-9} \mathrm{~s}$ \\
\hline Differential gain in active region & $3 \times 10^{-16} \mathrm{~cm}^{2}$ \\
\hline Differential gain in absorption region & $1.2 \times 10^{-15} \mathrm{~cm}^{2}$ \\
\hline Transparency carrier density in active region & $1.5 \times 10^{18} \mathrm{~cm}^{-3}$ \\
\hline Transparency carrier density in absorption region & $1.7 \times 10^{18} \mathrm{~cm}^{-3}$ \\
\hline Absorption and scattering loss in waveguide & $40-80 \mathrm{~cm}^{-1}$ \\
\hline Cavity length & $400 \mu \mathrm{m}$ \\
\hline facets reflection & 0.55 \\
\hline Effective group refractive index & 3.70 \\
\hline Carrier diffusion coefficient & $20 \mathrm{~s}^{-1} \mathrm{~cm}^{2}$ \\
\hline Approximate emission wavelength & $1.55 \mu \mathrm{m}$ \\
\hline Transverse confinement factor & 0.35 \\
\hline Linewidth enhancement factor & 4.0 \\
\hline
\end{tabular}

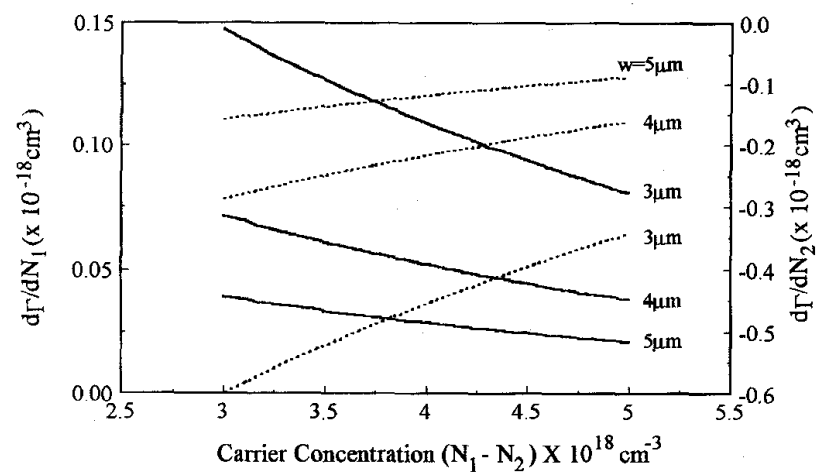

Fig. 2. Variation of differential confinement factor, $\partial \Gamma / \partial N_{1}$ and $\partial \Gamma / \partial N_{2}$, against carrier concentration, $N\left(=N_{1}-N_{2}\right)$ for stripe width equal to 3,4 , and $5 \mu \mathrm{m}$ (solid line $-\partial \Gamma / \partial N_{1}$, dotted line $-\partial \Gamma / \partial N_{2}$ ).

range of carrier concentration. This implies that our numerical study of $\partial \Gamma / \partial N$ is consistence with our analytical analysis.

\section{CONCLUSION}

The influence of lateral field on the dynamic response of semiconductor lasers is clarified. This is caused by the carrier dependence of lateral field distribution and this lateral effects can be characterized by the equivalent differential confinement factor, $\partial \Gamma / \partial N$. Furthermore, it is found that for lasers with strong index guided, negative value of $\partial \Gamma / \partial N$ is a must, however, for gain-guided devices with suitable stripe width and material parameters, $\partial \Gamma / \partial N$ is a positive quantity. Therefore, gain guided devices can perform better dynamic response than the strong index guided lasers.

\section{REFERENCES}

[1] G. P. Agrawal and N. K. Dutta, Long-Wavelength Semiconductor Lasers. New York: Van Nostrand Reinhold Company, 1986, and the references inside.

[2] K. Petermann, Laser Diode Modulation and Noise. Norwell, MA: Kluwer Academic, 1988.
[3] A. Yariv, Quantum Electronics, third edition. New York: John Wiley \& Sons, 1987.

[4] M. Yamada, "A theoretical analysis of self-sustained pulsation phenomena in narrow stripe semiconductor laser," IEEE J. Quantum Electron., vol. 29, no 5, pp. 1330-1336, 1993.

[5] M. J. Adams, An Introduction to Optical Waveguides. New York: Wiley, 1981.

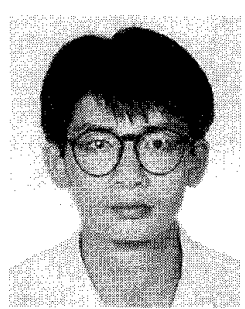

S. F. Yu received the B.Eng. degree (with Departmental prize) in electronic engineering from London University, University College, England, in 1990 and Ph.D. degree in Optoelectronics from Cambridge University, Robinson College, England, in 1993.

He is a Fellow and Honorary Scholar of Cambridge Commonwealth trust and holds a Croucher Foundation scholarship while studying for the Doctoral program. In 1994, he joined the Department of Electrical and Electronic Engineering, University of Hong Kong, where he is a Lecturer. His main research topics include wavelength selectivity of grating coupled waveguides, transient properties of semiconductor laser diodes, and design of optoelectronic integrated circuit. He currently conducts the development of high-performance semiconductor lasers for the application in high-speed communication systems. He also conducts the investigation of the influence of acoustic waves in semiconductor materials.

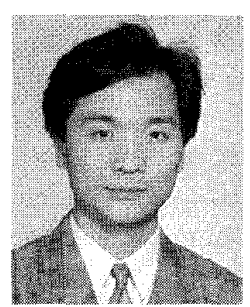

E. Herbert Li (S'87-M'88-SM'95) received the M.Phil. degree in applied mathematics and elec tronic engineering from the University of Hong Kong, in 1990 and the Ph.D degree in electronic engineering from the University of Surrey, UK, in 1992.

He worked for the Kirsten Aeronautical Laboratory, Seattle, WA (1979-1980). He joined K K. Engineering, Co., Hong Kong, as an Engineer (1981-1986), and Micro Systems, Hong Kong, as a Manager (1986-1988). He was a Member of Faculty at the City University of Hong Kong (1988-1990). He joined the National Ion Implantation Facility at the Department of EEE, University of Surrey, UK as a Research Project Leader (1990-1993). He is currently a Member of Faculty and the Leader of the Optoelectronics Team, which consists of a group of 15 researchers, at the Department of EEE, University of Hong Kong (1994-present). He also holds Visiting Faculty appointments at the University of Surrey, UK, and City University of Hong Kong, and is a Visiting Professor at Tsing Hua University, China. His current research interests are concerned with optoelectronic device fabrication, modeling and characterization, interdiffusion induced modification of quantum-well structures for the integration of optoelectronic devices, acousticoptic devices for slow-rate switching applications, photovoltaic devices, and the development of optical ATM switching systems. In 1994, he joined the department of Electrical and Electronic Engineering, University of Hong Kong, where he is a Lecturer.

Dr. $\mathrm{Li}$ is a member of the Hong Kong IEEE CASICOM Join Chapter Committee. He served on the Technical Program Committee of the IEEE TENCON '95, and on both of the Technical Program and International Advisory Committees of OECC'96, Japan. Dr. Li is an Editor of the International Journal of Optoelectronics and also an Honorary Life Advisor of Pi Mu Epsilon (US). He received the J. Langham Thompson Premium Prize in 1992 from the Institution of Electrical Engineers (UK) and the Distinguished Pioneering Projects Award in 1989 from HKCSS (HK). His biography is published in Who's Who in the World and Who's Who in Science and Engineering (Marquis, USA). Since 1990, he has published over 60 international technical papers and 2 monographs. 